\title{
Langue et identité. Légendes d'origine des Peuls
}

Language and Identity. Legends about the Fulani's Origin

Lengua y identidad. Leyendas de origen de los Fula

\section{Christiane Seydou}

\section{OpenEdition}

\section{Journals}

Édition électronique

URL : https://journals.openedition.org/clo/4714

DOI : $10.4000 /$ clo.4714

ISSN : 2266-1816

\section{Éditeur}

INALCO

\section{Édition imprimée}

Date de publication : 21 juin 2018

Pagination : 79-99

ISBN : 9782858313143

ISSN : 0396-891X

\section{Référence électronique}

Christiane Seydou, "Langue et identité. Légendes d'origine des Peuls », Cahiers de littérature orale [En ligne], 83 | 2018, mis en ligne le 20 juin 2019, consulté le 30 juin 2021. URL : http:// journals.openedition.org/clo/4714 ; DOI : https://doi.org/10.4000/clo.4714

\section{(c) (1) (8)}

Cahiers de littérature orale est mis à disposition selon les termes de la Licence Creative Commons Attribution - Pas d'Utilisation Commerciale 4.0 International. 


\section{Langue et identité. Légendes d'origine des Peuls}

Christiane SEydou

CNRS - LLACAN

Après son apport original et déterminant sur « l'ethnologie de la parole », Geneviève Calame-Griaule a consacré ses activités de recherche à justifier « l'introduction de l'étude de la littérature orale comme un chapitre de l'ethnolinguistique ${ }^{1} \gg$; laissons donc les Peuls lui offrir ici une confirmation on ne peut plus éclatante avec leurs légendes d'origine qui donnent comme créatrice même de leur ethnie, la langue.

En Afrique, dans l'aire où, tout au long de leur histoire, se sont répandus les Peuls, ont eu lieu tant de brassages et de métissages de populations et s'est imposée une telle diversité de conditions (écologique, économique, sociologique, culturelle, religieuse...) que l'identité peule, si on cherche à la cerner dans la réalité, est en fait protéiforme ; et, bien souvent, diluée dans les méandres de la vie et les aléas des situations historiques, elle n'existe qu'à l'état virtuel, à travers un discours décalé bien qu'encore unitaire faisant - tantôt explicitement, tantôt tacitement - référence au pulaaku, manière d'être et de se comporter censée définir cette communauté.

Si les Peuls font si souvent appel à ce pulaaku, sans doute est-ce le signe qu'il existe chez eux un besoin de défendre cette notion d'identité contre la conscience qu'ils ont, eux-mêmes, de sa labilité au contact d'un environnement tant humain qu'écologique sans cesse différent, au fil de leurs déplacements et de leurs établissements successifs.

1. Calame-Griaule, 1977, p. 22. 
Et s'il est incontestable que l'intervention de l'administration coloniale a une responsabilité dans la question des distinctions ethniques, il est tout aussi évident que - en tout cas en ce qui concerne les Peuls - c'est leur propre discours qui a pu susciter chez les Européens cette vision obsessionnelle de leur spécificité. En fait foi l'abondance de témoignages de leur revendication d'une « foulanité », tant dans leur comportement social, psychologique, éthique... que dans leur discours « littéraire »; discours qui, inscrit forcément dans le binôme indissociable identité versus altérité ${ }^{2}$, manifeste l'affirmation de leur identité dans leur positionnement permanent comme l'étranger, l'Autre, par excellence. C'est ce que décrit une abondante série d'études parues dans la dernière décennie du $\mathrm{XX}^{\mathrm{e}}$ siècle $^{3}$.

Cette distance par rapport à autrui et au monde, cette revendication d'altérité pour eux-mêmes est sans doute imputable à leur statut originel de migrants dans la plupart des régions où ils se sont implantés, situation qui a dû leur imposer diverses stratégies de défense et une attitude de repliement qui n'ont fait qu'exacerber leurs modalités intimes de représentation identitaire; sans doute est-ce là, aussi, la raison de l'émergence de nombreuses légendes d'origine où se révèle cette préoccupation d'explication des différences distinctives non seulement entre les Peuls et les autres, mais, à l'intérieur de l'aire peule, entre ceux sédentarisés et ceux restés nomades et, à l'intérieur de la société peule, entre eux et les différentes catégories socio-professionnelles indispensables à toute vie communautaire.

Ainsi, à travers ces légendes dont l'existence même témoigne de cette « étrangeté » ressentie et revendiquée, se dessinent les contours d'une identité à géométrie variable, selon le niveau envisagé. La variabilité des versions rend compte en effet de l'évolution des représentations qu'un peuple peut avoir - ou vouloir - donner de lui-même, en fonction des circonstances, de son devenir historique, de ses empreintes idéologiques successives. C'est là, ce que nous nous proposons d'explorer.

2. Ce sujet a été en partie abordé dans un autre article. SEYDOU, 2014.

3. Cf., entre autres, Cabiers d'études africaines, no 133-135 ; BRUIjn \& van DijK (dir.), 1997 ; Bierschenk \& Le Meur (dir.), 1997 ; Botte, Boutrais \& Schmitz, 1999, en particulier, l'article d'E. Boesen; et, dans Diallo \& SChlee (dir.), 2000, l'article de Martine Guichard. 
Si nous négligerons ici le déferlement de récits concernant l'origine « historique » des Peuls ${ }^{4}$ - origine qui a alimenté les hypothèses les plus variées et les plus débridées selon les auteurs et les époques voire les options politiques -, au profit de ces légendes populaires, c'est que les tentatives de réponses qu'apportent celles-ci à l'interrogation sur eux-mêmes qui semble tarauder les Peuls, traduisent plus légitimement les points nodaux autour desquels se cristallisent leurs représentations communes, leurs véritables points d'ancrage identitaire.

\section{Langue et pastoralisme}

De l'ensemble des légendes ${ }^{5}$ recueillies nous ne retiendrons en premier que deux motifs qui apparaissent significatifs du fait de leur coalescence mais aussi des modifications de leur exploitation selon le contexte historique ou idéologique : le pastoralisme et la langue. On verra que ces deux motifs, intimement liés dans les légendes familières aux Peuls pasteurs, se disjoignent progressivement chez les Peuls sédentaires au point que, dans les versions islamisées des Peuls citadins, celui du pastoralisme finit par disparaître au profit de celui de la langue.

Voyons en premier la légende la plus simple rapportée par un nomade bodaado ${ }^{6}$ :

1a. (résumé) Deux orphelins, un frère et une sœur, en allumant un feu au bord d'un fleuve, virent un jour une vache sortir de l'eau ; en éloignant leur feu de la rive, ils attirèrent progressivement tout un troupeau et c'est ainsi qu'ils devinrent pasteurs. Le frère et la sœur se marièrent et eurent deux enfants qui furent à l'origine, l'aîné, des Peuls, le cadet, des Wodaabe (i.e. des Peuls nomades du Niger), leur différenciation ne tenant qu'à la longueur des cornes de leurs vaches; en effet, une fois grands, sur l'injonction de leur père, ils choisirent, l'aîné, les vaches aux cornes courtes, le cadet, celles aux cornes longues.

4. Pour simplifier ce rappel, on peut en avoir un aperçu à travers les textes et les sources cités dans Tauxier, 1937 ; Hama, 1968, etc.

5. Précisons que l'ordre de présentation de ces légendes ne doit en rien à une chronologie supposée ; l'ensemble des versions étudiées est pris comme un simple corpus de données dont les variantes peuvent être éclairantes, indépendamment de leur émergence dont toute chronologie est impossible à établir.

6. Dupire, 1962, p. 29-30. 
Une version plus précise (1b) explique que les deux enfants (le frère et la sœur) se sont retrouvés isolés dans la brousse, livrés à eux-mêmes, parce que, s'étant mis à parler, un beau jour, une langue inconnue de leurs parents, ceux-ci, effrayés, les chassèrent les prenant pour des djinns. Ainsi est-ce l'apparition ex nibilo d'une nouvelle langue qui serait à l'origine de leur rapport avec le pastoralisme; et ce sont ces deux données qui, d'emblée, apparaissent ici comme fondatrices de l'identité peule.

\section{Langue et islam}

Avec l'instauration de l'islam, ont fleuri à profusion d'autres légendes faisant référence, cette fois, à un métissage originel arabo-africain, considéré assurément comme plus flatteur qu'un inceste originel (évidemment honni dans une société musulmane) ! Si la filiation patrilinéaire arabe est stable, la filiation matrilinéaire, elle, varie selon la région de production de ces légendes : wolof, soninké, bambara, béribéri,...

Les légendes les plus simples reprennent la précédente ${ }^{7}$ :

1c. (résumé) le père des deux enfants est un Arabe, voire un prophète (!) venu islamiser le pays et le garçon qui est muet se met un jour à parler à sa sœur dans une langue inconnue ; constatant cette anomalie, le père effrayé chasse les enfants qui, désormais, vivent tout seuls au bord de l'eau, puis voient en sortir une vache et adoptent le pastoralisme.

Ici intervient un élément qui va s'imposer dans les versions islamisées : le mutisme préalable à l'apparition de la nouvelle langue, ce qui en accentue l'aspect miraculeux ; et, autre particularité, si le motif du pastoralisme est encore présent, en revanche, celui de l'inceste est évacué.

Comme on le voit, d'après ces trois récits, la différenciation se fait en deux étapes : outre la scission linguistique et familiale (le père exclut ses enfants locuteurs d'une autre langue que la sienne, en $1 \mathrm{~b}$ et $1 \mathrm{c}$ ) qui donne naissance aux Peuls, intervient, à la génération suivante, une seconde scission - moins radicale, celle-là - par la primogéniture (aîné, Peul et cadet, Bororo, en 1a) qui donne naissance, cette fois, à deux groupes distincts : les sédentaires et les nomades (les Bororo) ; cette différenciation résulte, dans la première version (1a), de la seule 
distinction phénotypique (longueur des cornes des vaches : courtes choisies par l'ainé, longues choisies par le cadet).

Dans les légendes plus influencées par le contexte islamique, le motif de la langue inconnue parlée par un muet s'impose peu à peu au détriment de celui du pastoralisme qui disparaît totalement, comme le montre une version recueillie en Guinée $^{8}$ :

2a. (résumé) un Arabe vient d'Égypte avec une troupe islamiser les Wolofs; l'un de ses hommes épouse la fille du roi et ils ont quatre fils dont le troisième est muet. Un jour la mère entend le muet parler dans une langue qui lui est inconnue et dire au dernier-né qui pleure : « deyyu, neene aray joonin, a muynay » (calme-toi, maman va venir tout de suite, tu vas téter). Elle court avertir son mari, marabout, à la mosquée ; celui-ci consulte Le livre des langues (de Mahomet) et y trouve qu'une population de teint clair et parlant une langue nouvelle, dominerait une partie du monde. Il parcourt alors le monde pour annoncer que la langue prédite par le Prophète était découverte. Le muet apprend sa langue à ses frères et ils se dispersent : les deux aînés vont au Massina, le troisième au Foûta-Djalon et le dernier vers le Moyen-Niger et le Tchad.

Notons que, contrairement aux premières légendes citées, l'apparition de la langue inconnue est interprétée on ne peut plus positivement et qu'elle entraîne non pas le rejet des enfants hors de leur famille, mais au contraire le départ de leur père pour répandre la bonne nouvelle. Au silence concernant la vocation pastorale, point de ralliement - devenu purement idéel - pour la diaspora peule, s'oppose ici l'évocation de la dispersion géographique - bien réelle - des Peuls, chaque frère étant présenté comme responsable de leur installation dans les régions où on les trouve actuellement.

Une autre version $(2 b)$, très proche de celle-ci, donne encore aux Peuls un père arabe ou maure ; mais c'est, cette fois, dans le Coran même que celui-ci découvre que le Prophète aurait prédit la domination d'une partie du monde par des gens parlant une langue inconnue. Il part, abandonnant ses quatre fils chez « les Noirs » qui les chassent excepté l'ancien muet, Diallo, qui a appris la langue à ses frères ; lesquels partent au Massina et au pays haoussa ${ }^{9}$.

8. MAdrolle, 1895, p. 294-298.

9. ARCIN, 1907, p. 227. 
Nous retiendrons une dernière version rapportée par Robert Arnaud ${ }^{10}$, sous le titre de « la singulière légende des Soninké » :

3. (résumé) Oukbatou, venu du Yémen, épouse la fille du roi soninké ; et les quatre fils nés de cette union portent les noms de Diallo, Bâ, Sô et Bari (noms des quatre clans peuls de base). Les quatre enfants se mettent à parler une langue qui n'est ni l'arabe ni le soninké ; leur père se souvient que Mouhammad, avant sa mort, aurait déclaré : « il surgira après moi dans les contrées de l'Ouest, une tribu très fervente et très fidèle et que j'aime beaucoup par avance. Cette tribu parlera une langue qui n'est semblable à aucune autre. » Il décide de repartir au Yémen pour vérifier la chose et laisse sa femme ; celle-ci, après avoir attendu vainement son retour, finit par épouser Diaoua, le captif d'Oukbatou, et en a un fils, Dabi, qui sera l'ancêtre des Diawambé (groupe associé aux Peuls). Une fois adultes, les quatre premiers fils (qui parlaient le peul) vont à la Mecque où leur foi est mise à l'épreuve (ils accepteraient la perspective d'avoir la tête tranchée pour aller voir le Nabi au ciel). Et l'on découvre un écrit laissé par Mouhammad qui n'est rien d'autre qu'un vocabulaire de la langue parlée par ces pieux visiteurs. Ceux-ci repartent en Afrique de l'Ouest où ils sont rejoints par leur demi-frère, Diaoua, et les Diawambé.

Cette dernière version accentue encore davantage la relation établie entre la langue et l'islam dans la naissance du peuple peul ; comme on le voit, dans ces légendes, la réinterprétation islamique occulte totalement le motif de l'apparition du bovin, induisant un retournement total : la nouvelle langue n'est plus présentée comme responsable de la destinée pastorale des Peuls, mais comme la preuve de leur prédestination religieuse et d'un avenir politique privilégié. En outre, la nouvelle langue n'entraîne pas le rejet des enfants chassés par leurs parents effrayés par ce prodige ; c'est au contraire leur père qui, émerveillé par ce phénomène, part chercher la confirmation du miracle annoncé ; confirmation que les quatre frères trouveront, eux aussi, lorsque, partis à leur tour à la Mecque, ils auront pour preuve le livre en peul laissé par le Prophète. « Et l'on connut ainsi la langue peuhle créée par le Prophète lui-même ou créée par Dieu et

10. Cf. Arnaud, 1911 et 1912, p. 152-154 ; le résumé présenté ici reprend cette légende telle que la rapporte TAUXIER, 1937, ch. II. 
enregistrée par le Prophète ${ }^{11} \gg$ écrit Tauxier, citant une légende rapportée par Bernard Arnaud (1912).

Ainsi voyons-nous la langue changer totalement de statut : dans les premières légendes, inconnue de tous, elle est un moyen de communication intime si restreint qu'elle isole le frère et la sœur et les rejette hors du cercle familial et humain, les contraignant à un retour au monde animal de la brousse (avec le troupeau sorti de l'eau) et à un inceste primordial, imprimant ainsi une origine doublement « sauvage » au peuple peul. Avec la réinterprétation «islamisée », l'inconnu se mue en miraculeux, l'apparition ex nibilo en prophétie, et la stigmatisation tacite de l'origine incestueuse en glorification explicite d'un métissage arabo-africain sous le signe de l'islam.

Toutes ces versions recueillies auprès de Peuls sédentaires et citadins, témoignent d'un souci de justification de leur implication dans l'islamisation de l'Afrique de l'Ouest ; l'islam est d'ailleurs l'une des données sans cesse désignée comme constitutive du pulaaku; il vient donc s'ajouter à la première, la langue, en ajoutant à celle-ci une dimension religieuse, la projetant dans le monde de la prophétie et, par ricochet, désignant le peuple peul comme prédestiné à la communication et à l'extension du message religieux.

La disparition de la référence au pastoralisme signe une différenciation qui s'est instaurée chez les Peuls, de par leur histoire même, l'adoption de structures étatiques centralisées ayant accentué une distinction que traduisent bien, par ailleurs, les termes communément opposés de Fulbe Baleebe ou Fulbe wuronkoobe (Peuls Noirs ou Peuls de villages) et Fulbe Wodeebe ou Fulbe ladde (Peuls Rouges ou Peuls de brousse). L'appellation « Peuls Noirs » qualifie les Peuls sédentaires, urbanisés ; selon le contexte, ils sont considérés soit positivement parce que plus fidèles à l'islam que les Rouges, et même comme appartenant à l'aristocratie politique, soit, au contraire, péjorativement (par les Fulbe Wodeebe ) pour s'être éloignés de leur tradition pastorale originelle et s'être quasi assimilés aux autres populations de Balee6e (Noirs) cultivateurs; les Peuls Rouges, eux, sont les Peuls pasteurs nomades puis, plus généralement, ceux pratiquant encore l'élevage et non plus le nomadisme, mais seulement la transhumance ; et ces nomades sont, à leur tour, considérés par les sédentaires comme des broussards, d'un islam quelque peu approximatif: Wuronke e Pullo Bodeejo wanaa gootum, [un (Peul) villageois et un Peul Rouge ce n'est pas la même chose !].

11. TAuXier, 1937, ch. II. 
Cette distinction induit, à son tour, une mutation du thème des légendes d'origine, un glissement s'opérant alors, de l'origine des Peuls par rapport aux autres, à l'origine de ces deux catégories de Peuls.

\section{Peuls sédentaires et Peuls nomades}

Une fois admise l'existence des Peuls en tant que tels (par opposition à tous les autres désignés sous le terme de Haabe, sg. Kaado ${ }^{12}$ ), de nouvelles légendes cherchent à expliquer plus catégoriquement la différence entre les Peuls citadins ou sédentaires et leurs frères nomades. Pour cela, vont être sollicités des motifs déjà présents dans les légendes précédentes mais exploités différemment.

On a vu que les premières légendes évoquées (1a et 1b) donnaient aux Peuls et aux Bororo Wodaabe (Peuls nomades) une origine commune bien affirmée ; doublement frères puisque issus de l'union d'un frère et d'une sœur orphelins, ils ne sont différenciés que par l'ordre des naissances et, en 1a, par le choix des races bovines, l'aîné (Peul) ayant choisi les vaches à cornes courtes, le cadet (Bororo), les vaches à longues cornes ${ }^{13}$. La version (1c) introduit un père « prophète » et le motif de la langue inconnue; là encore, le départ du père entraîne celui des deux enfants qui, allumant un feu au bord de l'eau, attirent une vache et sont à l'origine de la domestication du bovin.

Que les enfants soient orphelins, séparés de leur père ou rejetés par leurs parents, la rupture de filiation verticale entraîne un resserrement des liens horizontaux de fraternité ; et leur isolement, qu'il soit dû à leur situation d'orphelins ou à leur communauté de langue, les exclut du reste du monde et les réunit d'autant plus; c'est ce qui les met, comme allant de soi, en situation de couple originel destiné à engendrer un nouveau peuple; et le recours à l'inceste n'appelle apparemment aucune réprobation explicite. Il semble même être là pour renforcer l'affirmation de l'origine commune des deux catégories de Peuls qui en sont issues.

Dans les versions franchement « islamisées », l'accent va, au contraire, être mis sur une filiation différente pour les distinguer de façon plus catégorique; pour cela, deux situations sont sollicitées : la bâtardise et l'inceste. On a vu l'inceste totalement évacué des versions islamisées qui présentaient les Peuls dans un environnement citadin et se distinguant des autres peuples non par une activité économique (le pastoralisme) mais par une langue, indice d'une prédestination

12. Haabe désignera ainsi les Dogon, les Songhay, etc. selon la région du Mali concernée. 13. Dupire, 1962, p. 29-30. 
d'ordre divin. Au contraire, lorsqu'il s'agit de distinguer ces Peuls de leurs frères nomades, ce motif infamant de l'inceste se trouve récupéré avec une virulence accrue, trahissant par là même l'origine de ces versions, vraisemblablement dues à des tenants d'un réformisme militant, comme en témoigne la référence à Ousman Dan Fodiyo dans la version nigérienne citée par Marguerite Dupire ${ }^{14}$, où les connotations négatives s'accumulent :

4a. (résumé) Une fille peule violée par un captif de son père en eut deux garçons et deux filles qui se marièrent entre eux. Le grand-père indigné par ces unions incestueuses, alla se plaindre auprès du Shaykh Ousmane Dan Fodiyo qui leur donna le nom de Wodaabe [Interdits].

L'origine doublement infamante donnée aux nomades fait ici référence et à la mésalliance (fille peule/captif) et au double inceste; et leur nom les stigmatise de par sa signification même et du fait qu'il leur est attribué par l'autorité religieuse la plus célèbre.

Cette réitération de l'inceste prend encore une autre forme narrative lorsque, cette fois (4b), il unit un Peul, orphelin de père, à sa propre mère puis à sa propre fille née de ce premier inceste ; cette fois, à la différence des versions précédentes, ces fautes sont involontaires, tout se passant à l'insu du coupable ; néanmoins, c'est l'enfant né de cette action maudite - aggravant l'inceste par la confusion des générations - qui est l'ancêtre des nomades : les Bororo Wodaabe ${ }^{15}$.

Dans une nouvelle série de légendes, le motif de l'inceste s'atténue au profit de celui de la bâtardise - sans toutefois gommer cette accumulation d'infamies, le père du bâtard n'étant pas un Peul ni même un humain mais un djinn - et, alors, le motif de la vache réapparaît, ces deux motifs se trouvant combinés pour mieux accentuer la spécificité du pasteur.

Une version rapportée par Marguerite Dupire conjugue, pour mieux opposer les deux groupes, les motifs de l'apparition de la langue peule et de la vache sortie de l'eau, avec celui de la filiation : ancêtre arabe venu de la Mecque, pour les uns, ancêtre génie sorti du fleuve, pour les autres ${ }^{16}$ :

5. (résumé) Oukoubou, venu de la Mecque pour islamiser le Soudan, a d'une épouse bambara, trois fils et une fille qui se

14. Dupire, 1962, p. 33.

15. Dupire, 1962, p. 33-34.

16. Dupire, 1962, p. 32-33. 
mettent à parler une langue inconnue. Un jour, la femme, partie se laver au bord du fleuve, est engrossée par un génie et en a un fils, Hakou. Chassée par son mari, elle retourne avec son bâtard dans sa famille ; celui-ci, ayant appris de sa mère qui était son père, va le trouver au fleuve et, suivant ses instructions, allume un feu qui attire les bovins hors de l'eau. Il donne des bêtes à ses demi-frères qui deviennent les ancêtres des Peuls et lui, épouse une femme bambara et est l'ancêtre des Bororo.

Nous avons là une recomposition des éléments présents dans les versions précédemment évoquées - père mecquois, langue inconnue, bovin attiré par un feu hors de l'eau - mais redistribués différemment pour une nouvelle projection idéologique destinée à justifier le statut des Bororo aux yeux des Peuls. La distinction entre les deux groupes se lit en effet dans une accumulation de justifications et déplace la fonction attachée aux motifs :

. filiation paternelle : père arabe (musulman)/père génie (païen)

- attribution de l'avènement de : langue nouvelle, aux enfants nés du couple femme bambara + mecquois/pastoralisme, à l'enfant né du couple femme bambara + génie de l'eau

- naissance de : Peuls, descendance d'Oukoubou (+ femme bambara)/Bororo, descendance de Hakou (+ femme bambara).

La conclusion de cette légende est inverse de celle de la toute première version. En effet, alors qu'en $1 \mathrm{a}$ et $1 \mathrm{~b}$, la séparation des deux groupes (issus d'une filiation doublement commune) était signifiée par le seul partage du troupeau ordonné par le père (image et affirmation d'une communauté culturelle originelle), ici, c'est le fils du génie qui, en faisant don à ses demi-frères utérins (fils d'un musulman et initiateurs de la langue) d'une richesse à lui seul octroyée (le troupeau, au fondement même de son statut économique, social et culturel), renoue avec eux au nom de cette parenté par le lait qui les unit, et établit une communauté basée non plus sur la filiation paternelle mais sur une activité commune passant par le bovin.

Ainsi se trouve rétablie entre les deux lignées la relation essentielle passant par filiation utérine et rapport au bovin, deux points d'ancrage fondamentaux de la culture et de la société peules. Et le rapport s'inverse : d'une fratrie originelle remaniée par l'intrusion d'éléments nouveaux mais partagés (langue et vache), on 
passe à une « famille recomposée » réunie par l'intrusion de ces mêmes éléments nouveaux mais attribués, cette fois, chacun à une partie distincte.

\section{Peuls et artisans « castés »}

Une dernière version ${ }^{17}$ attribuée à un groupe semi-nomade, introduit une nouvelle distinction en la déplaçant : de celle entre Peuls citadins ou sédentaires et Peuls nomades, on passe à celle entre Peuls et artisans « castés » :

6. (résumé) Une femme enceinte est abandonnée par son mari (ou son amant) qui, à l'insu de celle-ci, laisse dans la case un sachet contenant une poudre. L'enfant naît et grandit auprès de sa mère, victime de son statut de bâtard. Un vieillard de passage révèle un jour à la mère l'existence du sachet ; sur les instructions du vieillard l'enfant va faire en brousse, au bord de l'eau, une fumigation avec le contenu du sachet. Sort de l'eau un génie cyclope qui remet à l'enfant un livre en lui recommandant de suivre l'enseignement qui y est consigné, mais d'aller auparavant se vêtir de neuf (sans pantalon, toutefois ${ }^{18}$ ), de se procurer des ustensiles neufs et d'aller procéder à une deuxième fumigation avec les ingrédients prescrits dans le livre. L'enfant se rend chez le boisselier qui lui taille mortier et pilon, chez le tisserand qui lui confectionne de nouveaux vêtements, et chez le forgeron qui lui donne des braises. Il va cueillir les plantes et quérir les ingrédients indiqués dans le livre, il les pile dans son mortier et va procéder à la fumigation au bord de l'eau. Et voilà que, cette fois, ce sont les vaches qui sortent de l'eau. Il les ramène au village et va payer avec cette nouvelle richesse les artisans qui lui avaient fourni tout à crédit. Et c'est de là que datent les relations entre les Peuls et ces groupes d'artisans.

(On raconte par ailleurs que le livre remis par le génie contenait tous les secrets concernant la vie pastorale, les trajets de

17. Légende recueillie par mes soins auprès de Boubakar Bello et donnée par des Peuls semi-nomades de la région de Wouro-Guéladio, dans le Torodi, au Niger. Cf. SEydou, 1972, p. 215-233 (publication malheureusement entachée de coquilles, d'omissions de mots, etc.).

18. Notons que le large pantalon en tissu symbolise le citadin par opposition à la culotte de cuir portée par les nomades. 
transhumance mais aussi la science des remèdes et des procédés prophylactiques - dont sont crédités les Wodâbé - et qu'il fut transmis de génération en génération jusqu'au jour où, l'islam triomphant, les gens voulurent le brûler. Le génie l'envoya chercher et l'envoyé disparut avec lui dans les eaux. On dit qu'il était écrit dans une langue que connaissait le premier Peul et que comprenait la première vache. Les maîtres initiateurs en auraient conservé la mémoire et les devins qui pratiquent l'hydromancie, y lisent leurs prédictions.)

Cette légende semble refléter la dualité culturelle qui imprègne le monde du semi-nomade dont les deux pôles restent la vache et le Livre (c'est-à-dire l'islam); toutefois elle adopte un point de vue « décomplexé » par rapport aux versions « islamisées » précédentes et, en y réintégrant le motif du livre propre aux Peuls citadins - d'un islam orthodoxe -, elle le réajuste à la vision des Peuls pasteurs nomades - d'un islam moins strict.

Cette dernière version nous permet en effet, avec son originalité, de revisiter la circulation de chaque motif et les transformations qui en découlent.

\section{Motif de la rupture de filiation}

Il semble évident que l'apparition d'une nouvelle ethnie ait pour justification élémentaire la rupture d'une filiation pour pouvoir en générer une autre ; cette rupture prend plusieurs formes selon la vision sous-jacente :

. a) elle est le fait

- des deux parents qui rejettent fils et fille, pris pour des génies (1a, 1b),

- mais plus souvent du seul père qui, soit les chasse (1c), soit part (2a, $2 \mathrm{~b}, 3$ et 6), soit renvoie sa femme engrossée par un génie (5).

b) elle est provoquée

- soit (le plus fréquemment : 1b, 1c, 2a, 2b, 3 et 5) par l'apparition d'une nouvelle langue inconnue, qui instaure une double séparation du côté agnatique, entre Arabes et Peuls, et du côté utérin, entre Peuls et autres Africains, 
. soit (5 et 6) par la bâtardise aggravée par l'inceste (4a, 4b) qui instaure, dans ce cas, une double séparation, au niveau de la filiation générationnelle et de la collatérale,

. ou encore (6) par l'absence de père, entraînant une équivalence explicite entre orphelin et bâtard («femme abandonnée par mari ou amant »).

Ce motif tel qu'il est traité et exploité dans le circuit narratif de ces légendes, ne laisse pas d'évoquer la superposition persistante dans la société peule de la filiation utérine ou «parenté par le lait » et de la filiation patrilinéaire privilégiée par l'islam. On a vu comment, dans la première version, à la rupture totale de la filiation verticale s'opposait le resserrement des liens horizontaux de fraternité et son aboutissement extrême dans l'inceste ; sans aller jusque-là, dans les autres versions, c'est finalement la parenté par le lait qui l'emporte :

- l'absence du père déléguant à la fratrie la responsabilité d'engendrer les lignées peules : les quatre clans $(2 \mathrm{a}, 3)$,

- la distinction de statut des pères donnant naissance aux Peuls et assimilés, les Diawambé (3),

- la différence de nature des géniteurs, conférant à la seule fraternité par le lait, la communauté de filiation liant Peuls et Bororo : en 5, il est précisé que, la mère ayant été renvoyée dans sa famille par son mari, le bâtard est élevé dans sa famille maternelle avant de retrouver son père génie, d'en obtenir les bovins et, grâce à ceux-ci, de rétablir la filiation collatérale avec ses frères utérins.

\section{Motif de l'apparition du bovin}

La destinée pastorale des Peuls est l'une des constantes les plus résistantes dans leurs représentations identitaires qui persistent même dans les aires du monde peul où leur sédentarisation a culminé avec l'instauration d'états centralisés et entraîné l'abandon de l'élevage; dans nos légendes, elle est consécutive à la rupture de filiation, mais sous deux aspects :

- a) la rupture générationnelle totale $(1 \mathrm{a}, 1 \mathrm{~b})$ met le frère et la sœur en situation de novateurs absolus : l'avènement du bovin, provoquée par le feu qu'ils allument au bord de l'eau, prend valeur d'épiphanie et leur 
attribue la responsabilité entière de leur destinée pastorale. Toutefois l'interprétation « islamisée » de cette version atténue cette rupture, voire la nie : le père, ayant entendu les enfants parler cette langue inconnue, part vers l'eau et, lorsque les enfants partent à leur tour, il est dit que c'est pour suivre leur père (1c) ; et c'est ainsi que, pendant qu'ils dorment seuls au bord de l'eau, une vache en sort attirée par le feu. Ici tout se passe donc comme si c'était leur père « prophète » qui, par son départ même, les avait guidés vers leur destinée pastorale, assumant ainsi subrepticement la continuité de sa relation paternelle. Dans ce cas, l'apparition du bovin n'est pas, en soi, ce qui établit une distinction entre les deux catégories de Peuls, celle-ci n'étant même pas évoquée ici, ou bien ne dépendant, comme dans le premier cas, que du critère de choix entre races bovines (longues cornes/cornes courtes) ; dans toutes ces versions, le pastoralisme est bien la situation originelle commune des enfants du couple, à la différence des cas suivants.

b) substitution d'une filiation patrilinéaire non-humaine introduit le pastoralisme comme trait distinctif des seuls Peuls nomades, hérité de cette filiation surnaturelle liée au génie de l'eau ; et, dans ce cas :

- soit la différenciation s'établit entre les enfants du « prophète » et celui du génie par la révélation du bovin au seul « bâtard » qui, restant uni à ses frères par la filiation utérine, rétablit la relation de la fratrie en leur donnant des vaches (5) (notons que, si la langue des enfants légitimes est inconnue de leurs parents, elle ne l'est apparemment pas de leur frère utérin),

- soit, le « bâtard » est seul et le bovin l'amènera à établir un autre type de relation plus large, au niveau non plus parental, mais social, et non plus par le don, mais par l'échange (6).

\section{Motif du Livre}

Ce motif est celui qui présente le plus d'avatars dans ces légendes, selon qu'il $s$ 'inscrit dans une interprétation inspirée de l'islam ou du nomadisme :

- a) dans les premières, il est directement associé au motif de la langue nouvelle qui est alors magnifiée par le recours à une justification prophétique : annonce par Mouhammad d'une langue parlée « dans les contrées de 
l'Ouest, par une tribu très fervente et très fidèle et qu' il aime beaucoup par avance »; annonce que les locuteurs de cette langue « domineraient une partie du monde » ; évocation même de la découverte d'un dictionnaire de cette langue établie par Mouhammad... ; croyance que cette langue avait été créée par le Prophète ou « par Dieu même et enregistrée par le Prophète ${ }^{19}$ », au point que, en 6, cette langue et ce livre apparaissent presque comme un reflet mythique mais « laïcisé » de la révélation du Livre sacré du Coran ;

b) la dernière version (6) donne en effet au motif du livre une tout autre dimension. Cette fois, c'est l'association du livre non plus à l'apparition de la langue nouvelle, mais à celle du bovin qui est mise en scène ; l'apparition de la langue nouvelle reste implicite : il apparaît en effet évident que l'enfant, non seulement a la connaissance de la langue dans laquelle est écrit le livre, mais, mieux encore, sait la lire ; le motif est ainsi à peine effleuré, comme allant de soi : l'enfant comprend le livre qu'il lit d'emblée pour en suivre les instructions. En revanche, livre et vache sortent de l'eau et sont, de la sorte, intimement associés à l'élément aquatique et à un personnage mythique (borgne ou cyclope) comme en témoignent de multiples gloses ${ }^{20}$. Et ce livre apparaît comme une sorte de Bible du pasteur, un pendant du Livre sacré des musulmans : en effet, comme celui-ci, il est, dans cette dernière version, le fruit d'une révélation et constitue une Somme, portant en lui, pour qui sait le lire, la totalité des connaissances nécessaires au pasteur.

Pour nous en tenir à cette légende, nous constatons que, cette fois, même si la filiation paternelle est rompue physiquement, il n'en reste pas moins qu'elle est maintenue au plus haut point par la responsabilité du père dans la destinée de l'enfant : le sachet de poudre qu'il laisse, à l'insu de sa femme, est le medium de l'héritage paternel, qui déclenchera tout le processus de la révélation; le génie sorti de l'eau apparaît en effet comme l'avatar de ce père, et le livre qu'il tient, comme celui du Livre sacré ou de celui en langue peule prétendument attribué au Prophète. On a ici une image inversée du père des versions islamisées : dans celles-ci, le père, arabe musulman, présent au début, part chercher dans les livres le secret de la langue nouvelle parlée par ses enfants... et la vache disparaît de ces versions ; dans celle-là, le père, absent au début, réapparaitt sous les traits du génie

19. Voir Arnaud, 1912, p. 142-154.

20. Voir Seydou, 1972, p. 228-232. 
aquatique et porteur du livre, source de ce qui fera, avec l'apparition de la vache, le Peul originel : le pastoralisme.

Toutefois, une péripétie narrative vient ici ajouter un nouveau motif qui témoigne, là encore, de la vocation des légendes à justifier l'actualité du vécu. Il s'agit d'expliquer non plus les relations entre Peuls nomades et Peuls sédentaires, mais celles entre Peuls (caractérisés par le seul trait distinctif du pastoralisme) et classes socio-professionnelles endogames regroupées habituellement sous le terme approximatif de « gens de castes ». Pour expliquer la naissance de cette structure dans la société peule, il existe au Sénégal un très beau récit, le Fantang ${ }^{21}$, qui donne à tous, Peuls et « castés », une origine commune; il s'agit en effet de trois frères et leur différenciation s'établit à partir des occupations choisies par chacun. Dans notre légende, au contraire, les deux catégories sociales sont d'emblée étrangères l'une à l'autre : les artisans sont déjà là et c'est, avant toute chose, l'obligation pour le nouveau venu d'engager une relation avec chacun d'eux qui est évoquée pour inaugurer l'insertion de l'élément nouveau, lié à la nature sauvage, dans un monde socialement organisé qui lui est étranger. En effet l'injonction du génie adressée à l'enfant de procéder en homme nouveau (habillé de neuf et avec des ustensiles neufs) à la fumigation indiquée dans le livre, amorce le processus de « domestication » du monde de la nature auquel il devra se livrer : l'apparition de la vache attirée hors de l'eau par un feu alimenté par des végétaux, lui alloue sa destinée de pasteur, médiateur entre les éléments naturels (eau, feu, végétal, animal) et celui de la vie « urbaine » mais aussi son insertion sociale en lui permettant, une fois le troupeau « domestiqué », d'entrer dans le circuit des échanges qui alimentent et structurent les réseaux socio-économiques; le pasteur donne aux artisans des vaches en échange des objets qu'il a obtenus d'eux pour pouvoir réaliser son appropriation du troupeau.

Ainsi est mise en scène l'interrelation existant entre Peuls et artisans « castés ». S'ils ne sont plus ici, originellement « frères », comme dans le Fantang, ils sont originellement liés par deux nécessités vitales réciproques : la fourniture, par les artisans, d'objets indispensables tant à la vie sociale (symbolisée ici par le vêtement) qu'à la production des biens de consommation (ici représentée par les objets « technologiques » : poterie et braise) et la fourniture, par les pasteurs, de ces biens de consommation-mêmes grâce à la domestication de la nature (ici représentée par la conjonction de végétal, feu, eau et animal). Et, cette fois, la légende n'a plus pour projet la justification d'une dissociation Peuls/autres ou Peuls sédentaires/Peuls nomades - mais, au contraire, celle d'une association - Peuls/artisans « castés ».

21. NDONGO, 1986. 
Dans cette dernière version de la légende recueillie dans une région où coexistent les deux situations - Peuls nomades et Peuls sédentaires -, la conjonction des motifs du Livre et de la Vache nous apparaît comme une tentative de réconcilier les deux points de vue, au prix toutefois d'une récupération du premier au bénéfice du second. Tout en accordant au Livre le rang suprême, puisque c'est de lui que dépend tout (apparition de la vache mais surtout connaissance de tout l'univers pastoral, utilisation des végétaux, etc.), c'est la capacité qu'a, seul, cet enfant de le lire et, ayant ainsi acquis la science du végétal (les ingrédients pour la fumigation), d'attirer à lui, grâce à la conjonction du feu et de l'eau, le monde animal, puis sa capacité à faire prospérer le troupeau, qui le consacrent comme ancêtre générateur des Peuls qualifiés ici - notons-le de cette seule dénomination (Peuls et nomades Wodaabe n'étant plus dissociés) et identifiés par leur seule qualité originelle commune de pasteurs.

Cette légende semble donc le fruit d'une contamination entre plusieurs données pour en faire un tout cohérent, en conciliant un thème mythique l'origine du bovin et sa domestication -, un thème culturel - le savoir pastoral : connaissance de la botanique, de la pharmacopée naturelle, des trajets de transhumance, des pratiques nécessaires à la vie du troupeau -, un thème sociologique - l'établissement des relations liant le Peul à la catégorie socioprofessionnelle des différents corps d'artisans -, un thème économique son intégration dans le système d'échanges assurant la cohésion sociale et l'équilibre économique.

À cela s'ajoute une adaptation du motif du livre qui, tout en le rejetant totalement de la sphère religieuse privilégiée dans les versions « islamisées », lui confère le rôle déterminant qu'a, dans celles-ci, la langue comme élément premier déclenchant tout le processus de l'avènement du peuple peul. En effet ce livre a la même fonction que la langue avec, toutefois - différence significative - le passage de la langue parlée, à la langue écrite; de même que la langue inconnue se révèle ex nibilo dans la bouche des enfants qui la parlent sans l'avoir apprise, de même le livre est miraculeusement offert à l'enfant qui le déchiffre sans y avoir été initié par le génie donateur, avatar de son géniteur; et, de même que la langue, en dissociant les enfants de leur filiation originelle, les révèle à leur nouvelle vocation ethnique et pastorale, ce livre opère les mêmes modifications dans le statut de l'orphelin : tout en lui assignant sa vocation pastorale, il lui octroie sa place dans l'espace social. De plus, en filigrane, ce recours à un livre révélé apparaît comme une revalorisation de la tradition pastorale qui s'avère parallèle, par cette référence à l'écriture, à la tradition des lettrés magnifiée par l'islam.

Ainsi les métamorphoses de cette légende d'origine des Peuls illustrent la malléabilité du matériau culturel, une même donnée étant filtrée différemment selon les contextes sociologiques ou historiques ; on voit ici comment le regard 
de chaque groupe privilégie un élément, un motif autour duquel se cristallise la structure du récit et sa signification, ou bien donne à un motif commun une orientation variable selon l'idéologie dominante. S'y retrouvent les principaux points d'ancrage du pulaaku, manière d'être conçue comme spécifique du Peul, dont pastoralisme et langue apparaissent comme les plus prégnants.

Toutefois, l'antériorité accordée à la langue, dans la majorité de ces légendes, comme fondatrice de l'ethnie et de sa vocation pastorale ne laisse pas de nous interroger sur les raisons de ce choix. Peut-être la situation des Peuls en Afrique suffit-elle à en éclairer la logique. On peut en effet trouver à cela une raison toute triviale : vu l'étendue de l'aire peule et la diversité des situations vécues par la diaspora peule selon les potentialités environnementales et au gré de l'évolution historique, la langue s'avère une possession commune qui, d'évidence, selon le binôme identité versus altérité, peut s'imposer comme premier élément d'identification tout à la fois en tant que bien inaliénable assurant une pérennité de communication aux groupes les plus éloignés, et comme lieu d'affirmation de soi face à la multiplicité des langues des voisins côtoyés. À cela s'ajoute le fait que le nomadisme originel des Peuls n'a pu qu'accentuer cet investissement dans la pratique de leur langue comme soutien identitaire et dans sa promotion comme matériau d'expression esthétique privilégié ; en effet, mis en contact avec des populations différentes, tout au long de leurs déplacements, les Peuls pouvaient ressentir en permanence leur propre spécificité autant par leur occupation que par leur langue ; en outre, leurs perpétuels déplacements leur interdisant tout encombrement, ils ne disposaient comme domaine et matériau d'expérimentation artistique que leur langue, d'où leur investissement dans une production littéraire aussi abondante que variée. Voilà qui explique, sans doute, la place privilégiée accordée à la langue comme fondatrice de l'ethnie dans leurs légendes d'origine.

Par ailleurs, à travers l'exploration de cette production langagière éminemment idéologique que sont les légendes d'origine, la jonglerie entre les trois motifs - langue, vache, livre -, bien représentatifs, pour les Peuls, des trois points d'ancrage principaux de leur identité, illustre l'inventivité nécessaire à toute population inscrite dans des destins si divers pour adapter ses repères afin de mieux résister au risque d'acculturation, et nous offre un exemple de cette dynamique de la culture qui a permis aux Peuls, en dépit de leur dispersion, de leur diversité, de leurs différences voire de leurs divergences, de se penser toujours comme restés eux-mêmes. 


\section{Bibliographie}

ARCIN André, 1907, La Guinée française : races, religions, coutumes, production, commerce, Augustin Challamel, Paris, $659 \mathrm{p}$.

ARnaud Robert, 1911, « La singulière légende des Soninké » in L'Islam et la politique musulmane française en Afrique occidentale française, Comité de l'Afrique française, Paris.

BAUMgaRdT Ursula (dir.), 2014, Représentations de l'altérité dans la littérature orale africaine, Karthala, Paris, $309 \mathrm{p}$.

Bierschenk Thomas \& Le Meur Pierre-Yves (dir.), 1997, Trajectoires peules au Bénin : six études anthropologiques, Karthala, Paris, 190 p.

Boesen Elisabeth, 1999, «Pulaaku. Sur la foulanité » in Botte Roger, Boutrais Jean \& Groupe D’études Comparatives Des Sociétés Peules (dir.), Figures peules, Karthala, Paris, p. 83-97.

Botte Roger, Boutrais Jean \& Schmitz Jean (dir.), 1999, Figures peules, Karthala, Paris, 539 p.

Bruijn Mirjam de \& Dijk Han van (dir.), 1997, Peuls et Mandingues : dialectiques des constructions identitaires, Afrika-Studiecentrum/Karthala, Leyde/Paris, $286 \mathrm{p}$.

Cabiers d'études africaines, $\mathrm{n}^{\circ}$ 133-135 (1994), L'archipel peul.

Calame-Griaule Geneviève, 1977, Langage et cultures africaines : essais d'ethnolinguistique, Maspero, Paris, $364 \mathrm{p}$.

Delafosse Maurice, 1972, Haut-Sénégal-Niger, 3 tomes, G. P. Maisonneuve et Larose, Paris, $426+428+314$ p.

Diallo Youssouf Hassane \& SChlee Günther (dir.), 2000, L'ethnicité peule dans des contextes nouveaux : la dynamique des frontières, Karthala, Paris, $255 \mathrm{p}$. 
Dupire Marguerite, 1962, Peuls nomades : Étude descriptive des Woöaabe du Sabel nigérien, Thèse de $3^{\mathrm{e}}$ cycle, Institut d'ethnologie, Paris, $338 \mathrm{p}$.

GuichaRd Martine, 2000, « L'étrangeté comme code de communication interethnique, les relations entre agropasteurs et paysans bariba du Borgou (Nord-Bénin) » in Diallo Youssouf Hassane \& SchleE Günther (dir.), L'ethnicité peule dans des contextes nouveaux : la dynamique des frontières, Karthala, Paris, p. 93-127.

Hama Boubou, 1968, Contribution à la connaissance de l'bistoire des Peul, Présence africaine, Paris, 366 p.

Madrolle Claudius, 1895, En Guinée, H. Le Soudier (Paris), Paris, 407 p.

Mollien Gaspard-Théodore, 1820, Voyage dans l'intérieur de l'Afrique, aux sources du Sénégal et de la Gambie, fait en 1818, par ordre du gouvernement français, Courcier, Paris, 339+319 p.

Monteil Charles, 1915, Les Khassonké: monographie d'une peuplade du Soudan français, Ernest Leroux, Paris, 528 p.

Ndongo Siré Mamadou (dir.), 1986, Le Fantang : poèmes mythiques des bergers peuls : textes de la tradition orale peule, Karthala, Paris, 204 p.

SEydou Christiane, 1972, « Une légende peule du Niger occidental, "la vache et le livre" » in Cabier des religion africaines, $\mathrm{n}^{\circ} 12$, vol. 6, p. 215-233.

Seydou Christiane, 2014, « De l'altérité d'un alter-ego dans la littérature orale peule du Massina (Mali) » in BAUMGARDT Ursula (dir.), Représentations de l'altérité dans la littérature orale africaine, Karthala, Paris, p. 225-251.

Tauxier Louis, 1937, Mours et histoire des Peuls, Payot, Paris, 419 p.

Résumé : les Peuls, conscients de leur altérité du fait même de leur situation originelle de pasteurs nomades qui les a mis en contact avec de multiples populations, ont élaboré de nombreuses légendes pour justifier leur apparition dans le monde. Trois motifs intéressants y dessinent leurs représentations identitaires : une langue nouvelle qui donne naissance à l'ethnie, l'apparition de la vache qui suscite leur activité distinctive, puis, avec l'islamisation, le recours à un métissage arabo-africain. C'est l'exploitation particulière de ces trois motifs, 
selon l'évolution historique et culturelle de chaque région de l'aire peule, qui sera analysée ici.

Mots-clefs : Peul, langue, vache, islam, identité, légendes d'origine.

\section{Language and Identity. Legends about the Fulani's Origin}

Abstract: because of their original situation as nomadic pastoralists, the Fulani came into contact with various populations; which made them aware of their otherness and, in order to justify their own appearance in the world, they have developped numerous legends. Three interesting motifs define their identity representations; a new language giving birth to their ethnicity, the emergence of the cow, which generated their distinctive activity and, lastly, with the advent of islamisation, the beginning of an arab-african mixing (metissage). This article will analyse the particular occurrence of these three motifs according to the historical and cultural evolution in the different regions of the Fulani area.

Keywords: Fula, language, cow, islam, identity, legends of origin.

\section{Lengua y identidad. Leyendas de origen de los Fula}

Resumen : Los Fula, conscientes de su alteridad por su situación original de pastores nómadas que los ha puesto en contacto con diferentes poblaciones, han elaborado numerosas leyendas para justificar su aparición.Tres motivos interesantes dibujan sus representaciones identidarias : una nueva lengua que da vida a la etnia, la aparición de la vaca que suscita su actividad particular, luego con la islaminación, el recurso a un mestizaje arabo-africano. Es la explotación particular de estos tres motivos, según la evolución histórica y cultural de cada comarca del área fula, la que va a ser analizada aqui.

Palabres calve : Fula, lengua, vaca, islam, identidad, leyendas de origen.

\section{Note sur l'auteur}

Christiane Seydou est agrégée de grammaire et diplômée de langue peule et de langue haoussa, directeur de recherche honoraire au CNRS, elle a consacré ses recherches à la langue et à la littérature des Peuls du Massina (Mali) qu'illustrent ses nombreuses publications concernant en particulier les genres épique et poétiques (pastoral et religieux). 